\title{
ZERO BIAS OPERATION OF THERMAL-PIEZORESISTIVE MICROMECHANICAL RESONATORS VIA INTERNAL ELECTROMECHANICAL MIXING
}

\author{
A. Rahafrooz and S. Pourkamali \\ Department of Electrical and Computer Engineering, University of Denver, Denver, CO, USA
}

\begin{abstract}
This work presents a novel operation technique for single crystalline silicon, thermal-piezoresistive resonators. This transduction technique takes advantage of internal electromechanical mixing of the resonant structure that eliminates the need for application of any bias current for resonator operation. In all previous works, a combination of DC bias and AC actuation signal was used for resonator operation. Here, it is demonstrated that such devices can exhibit resonant peaks in their electrical frequency response at half their mechanical resonant frequency by application of only a single AC actuation without the need for any power supply or biasing components. This allows engagement of such devices as electronic components with zero DC power consumption, which has been one of the main drawbacks of thermal-piezoresistive micro-resonators.
\end{abstract}

\section{INTRODUCTION}

MEMS resonators are emerging as modern frequency selective components gradually replacing the conventional quartz resonators in different markets [1]. The more popular classes of piezoelectric and electrostatic MEMS resonators have been welldeveloped over the past decade. However, the focus on activelytransduced micromechanical resonators, such as thermalpiezoresistive devices, has been quite limited. Such devices have received some attention over the past three years as their potential for high frequency and low power operation has been demonstrated [2]. In addition, they have the advantage of being electrically active devices (similar to transistors) providing unique capabilities such as self-sustained oscillation and self-Qenhancement [3-5]. Such capabilities along with their simple structure and low cost fabrication process make them suitable candidates for a wide range of RF applications. Moreover, unlike electrostatic resonators, thermal-piezoresistive resonators do not require any submicron transduction gaps. Therefore, they can be used as more robust and reliable sensors for particulate sensing applications [6] among others. All these benefits and potentials for improvement trigger interest in their further investigation.

Thermal-piezoresistive resonators are usually operated by applying a combination of DC and AC currents through their structure in order to thermally actuate them at the same frequency as the input signal. In addition, a DC current is required to turn the periodic change in the resistance of their sensing elements into a measurable fluctuating voltage to detect their mechanical vibration. In some thermal-piezoresistive resonators, different electrically isolated parts of the resonator are dedicated to thermal actuation and piezoresistive sensing $[7,8]$ while in others, thermal actuation and piezoresistive sensing are carried out simultaneously by a common part of the resonator [2]. In the former method, two independent bias currents pass through the actuating and sensing elements, whereas the later only requires a single DC bias current. Each of these methods has their own advantages and disadvantages but their common major drawback is the requirement of a DC bias current constantly burning valuable power, even when the resonator is at rest (not vibrating).

Here, a novel operation mechanism for monolithic thermalpiezoresistive resonators with common actuation and sensing element is demonstrated. The new technique takes advantage of the electromechanical mixing inside the resonator structure and waives the need for applying any bias current. In addition, this technique does not require any supporting electronic components such as bias resistors and capacitors. Therefore, it further decreases the overall power consumption and foot-print of the overall circuit. In order to fully investigate the zero bias operation of monolithic thermalpiezoresistive resonators, their conventional operation mechanism [2] is first described. Then, the new operation technique is explained and compared with the conventional one.

\section{OPERATION CONCEPT}

\section{A. Conventional Operation}

Figures 1a shows the required electrical setup to operate thermal-piezoresistive resonators using the conventional technique. In this method, upon application of a combination of AC actuation and DC bias currents $\left(I_{d c}+i_{a c} \cos \left(2 \pi f_{a}\right)\right)$ to the resonator structure an ohmic loss is generated, This ohmic loss has a squared relationship with the applied current and consists of three frequency components at $\mathrm{DC}\left(R_{A}\left(I_{d c}{ }^{2}+i_{a c}{ }^{2} / 2\right)\right)$, actuation frequency of the AC current $\left(2 R_{A} I_{d c} \cdot i_{a c} \cos \left(2 \pi f_{a}\right)\right)$ and twice the actuation frequency $\left(R_{A} i_{a c}{ }^{2} \cos \left(4 \pi f_{a}\right) / 2\right)$ where $R_{A}$ is the electrical resistance of the resonator. Due to the higher resistance of the two narrow beams in the middle of the structure, most of the ohmic power loss happens in them. The three resulting components translate into proportional temperature changes or fluctuations with the same frequencies. Each of the temperature components induces thermal stress in the mechanical structure and therefore mechanical forces at the same frequencies inside the narrow beams (Figures 2a). The alternating temperature of the beams causes their periodic expansion and contraction, actuating the mechanical structure at two different frequencies of $f_{a}$ and $2 f_{a}$. If the frequency of the $\mathrm{AC}$ input current $f_{a}$ matches the first in-plane extensional mode resonant frequency of the structure $f_{0}$, it starts resonating. During resonance, as the length and stress in thermal actuating elements fluctuates, their resistance is modulated due to the piezoresistive effect. Such periodic change in resistance $\left(r_{a c} \cos \left(2 \pi f_{0}\right)\right)$ of the thermal actuators is proportional to the vibration amplitude of the structure. Since a constant DC bias current is passing through the thermal actuators, this change in resistance is turned into a measurable $\mathrm{AC}$ output voltage $\left(I_{d c} \cdot r_{a c} \cos \left(2 \pi f_{0}\right)\right)$. In addition, the AC actuation current $\left(i_{a c} \cos \left(2 \pi f_{0}\right)\right)$ along with periodic changes in resistance of the piezoresistors gives birth to two voltage components at $\mathrm{DC}\left(i_{a c}, r_{a c} / 2\right)$ and twice the resonant frequency of the structure $\left(i_{a c} \cdot r_{a c} \cos \left(4 \pi f_{0}\right) / 2\right)$. Among the three output frequency components, only the one with the same frequency (green arrow in figure $2 \mathrm{a}$ ) as the input $\mathrm{AC}$ actuation current is picked up by the network analyzer and shows up in the transmission frequency response.

In terms of power consumption, in order to create a fairly constant DC bias current, the resonator is connected in series with two $(\sim 5 \mathrm{X})$ larger resistors with the whole combination connected to a power supply. The more appropriate way is to use a DC current source. However, both of these configurations consume some DC power which is usually larger than that of the resonator. 
a)

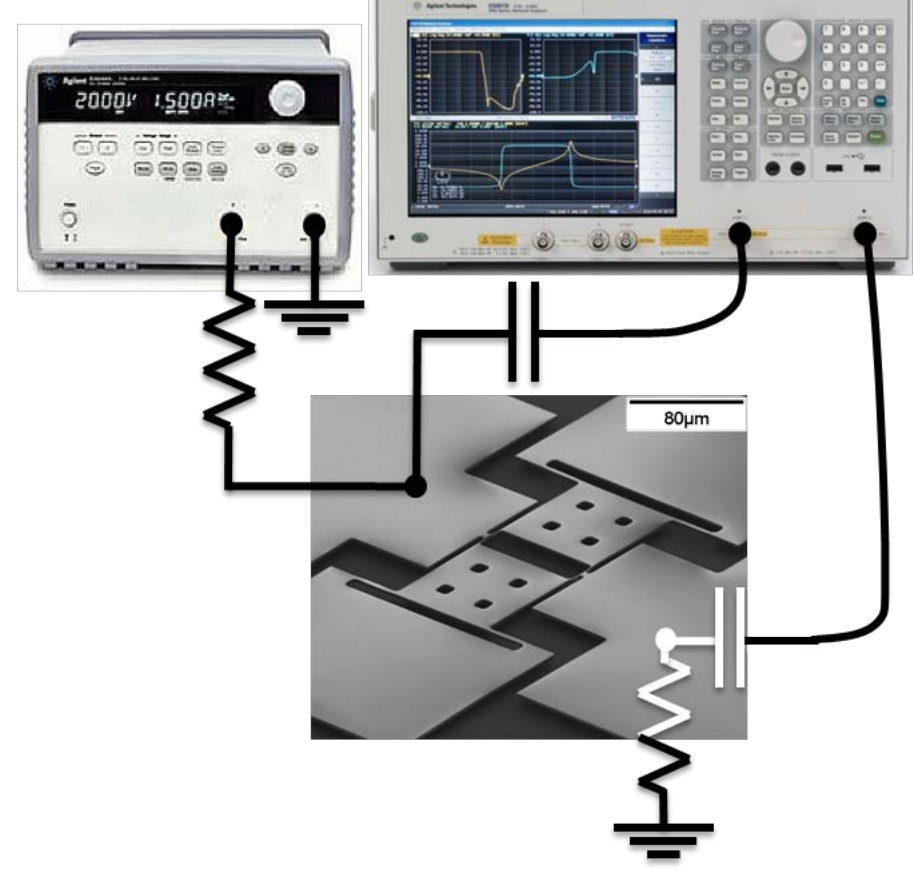

b)

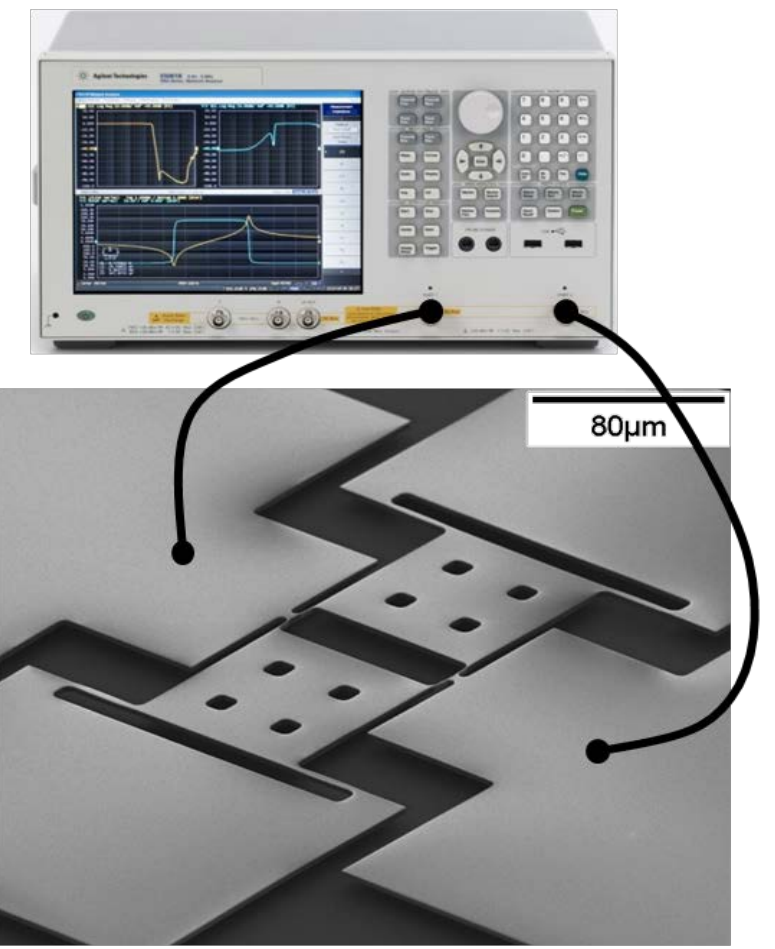

Figure 1: Schematic diagrams of the electrical connections for operation of thermal-piezoresistive resonators by application of a) a combination of DC and AC voltages/currents using a DC power supply, a network analyzer, two bias resistors and two decoupling capacitors and b) a pure AC signal at half the mechanical resonant frequency of the resonator by directly connecting the two terminals of the network analyzer to the resonator pads. In both cases, the network analyzer measurers the transmission data and the scanning electron microscope view is a fabricated $3.47 \mathrm{MHz}, 3.5 \mu \mathrm{m}$ thick resonator tested to demonstrate the concept.

\section{B. Zero Bias Operation}

In the new configuration, the resonator is directly connected to the $\mathrm{AC}$ source (network analyzer in this case) (Figure 1b). Upon application of the $\mathrm{AC}$ actuation current $\left(i_{a c} \cos \left(2 \pi f_{a}\right)\right)$ to the structure, the generated ohmic power $\left(i_{a c}{ }^{2}\left[1+\cos \left(4 \pi f_{a}\right)\right] / 2\right)$ and its resulting temperature and thermal expansion fluctuations will have two frequency components; one at DC and the other at twice the applied input frequency. If the frequency of the input $\mathrm{AC}$ current is half the resonant frequency of the resonator, the thermally generated force will have a component at the right frequency $f_{0}$ to excite the structure. During the mechanical vibration and due to the piezoresistive effect there will be periodic changes in the electrical resistance of the resonator at the same frequency as the resonant frequency of the structure $\left(r_{a c} \cos \left(2 \pi f_{0}\right)\right)$. Such resistance fluctuations are then mixed by the actuation current that has half the resonant frequency $\left(i_{a c} \cos \left(\pi f_{0}\right)\right)$. This modulation gives birth to two frequency components at $f_{0} / 2$ and $3 f_{0} / 2$ (Figure $2 \mathrm{~b}$ ). The two output voltage $\left(v_{\text {out }}\right)$ frequency components can be derived to be:

$$
\begin{aligned}
& v_{o u t}=r_{a c} \cos \left(2 \pi f_{0}\right) \cdot i_{a c} \cos \left(\pi f_{0}\right)= \\
& \frac{r_{a c} \cdot i_{a c}}{2}\left(\cos \left(\pi f_{0}\right)+\cos \left(3 \pi f_{0}\right)\right)
\end{aligned}
$$

Therefore, due to this internal electromechanical mixing there will be an electrical component at half the resonance frequency changing the transmission characteristics of the resonator as seen by the electrical components connected to it at resonance. As a result, in this technique, instead of having a resonant peak at resonant frequency, a peak appears in the electrical frequency response at exactly half the mechanical resonant frequency.
Figure 2 compares the operation details of both transduction mechanisms using block-diagram demonstration of the thermoelectromechanical model of such resonators [2]. It shows all the generated frequency components of the main physical parameters involved in the resonator transduction. Although in both techniques the structure resonates at its resonant frequency, but during zero bias operation the frequency of the input and output electrical signals is half the mechanical resonant frequency of the resonator. Moreover, unlike the conventional operation, in zero bias operation the peak amplitude is dependent on the input signal amplitude making it nonlinear.

\section{DEVICE FABRICATION AND MEASUREMENT RESULTS}

In order to show the new operation concept a dual-plate thermal-piezoresistive resonator was used. The resonator has been fabricated on a low resistivity N-type SOI substrate with the device layer thickness of $3.5 \mu \mathrm{m}$ using a single mask SOI MEMS process. Its thermal actuators were aligned along the $<100>$ crystalline orientation of silicon to obtain the highest piezoresistive coefficient [10] and provide a stronger transduction. Figure 1 shows the scanning electron microscope view of the fabricated $3.47 \mathrm{MHz}$ resonator along with the required electronic connections for both operation mechanisms. In conventional configuration, two bias resistors of $330 \Omega$ and two de-coupling capacitors of $0.1 \mu \mathrm{F}$ were used. DC and AC signals were supplied by a digital power supply and a network analyzer respectively. In both cases the transmission $\mathrm{S}_{21}$ parameters were recorded by the network analyzer.

Figure 3 demonstrates the transmission frequency responses of the $3.47 \mathrm{MHz}$ resonator obtained using both operation 
a)

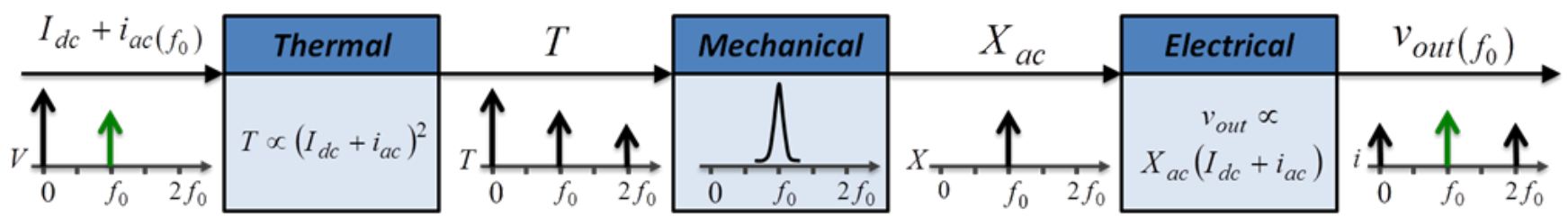

b)

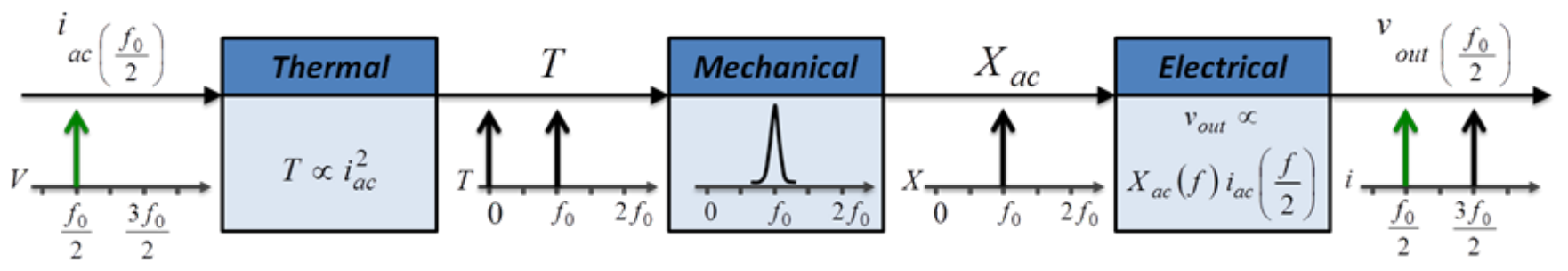

Figure 2: Block-diagram demonstration of thermal-piezoresistive operation of a resonant structure. a) Operation with DC bias: Upon the application of a combination of DC and AC currents, the generated power has a component at the same frequency as the AC input current that can actuate the mechanical structure into resonance. The mechanical vibration of the resonator modulates the resistance of the resonator due to the piezoelectric effect. The fluctuating electrical resistance in the resonator is then modulated by the DC current leading to an output component with the same frequency as the input $A C$ current that can be detected by the network analyzer. b) Operation without DC bias: By applying an AC current at half the resonant frequency of the resonator, there will be a power component at the resonant frequency of the resonator. This power in turn generates a thermal force at the same frequency that actuates the mechanical structure into resonance. During the vibration, the fluctuations of the resistance of the resonator with the resonant frequency of the resonator are modulated by the AC input current providing two different frequency components at the output. The component with half the resonant frequency can be detected. In this case the network analyzer shows a resonant frequency peak at exactly half of the resonant mechanical frequency of the structure. Green arrows show input/output frequency pairs with the same frequencies that are being generated or picked up by the network analyzer to measure the transmission data. In this figure, variables $I_{d c}, i_{a c}$ and $v_{\text {out }}$ are the DC bias current, input $A C$ voltage, input $A C$ current, and $A C$ output voltage of the resonator, $f_{0}, T$ and $X_{a c}$ are the resonant frequency, temperature and mechanical vibration amplitude of the resonator respectively.

techniques. As expected, the frequency response attained from the zero-bias technique (Figure $3 b$ ) shows a resonant peak at exactly half the mechanical resonant frequency of the resonator (Figure 3a). Due to the negative piezoresistive coefficient of the structural material (N-type silicon) [10] in both cases the resonant peaks are pointing downwards. In conventional operation, power of the network analyzer was kept at $0 \mathrm{~dB}$ and the transmission data was recorded at different DC bias currents. On the other hand, in zero bias operation, transmission data were recorded at different applied

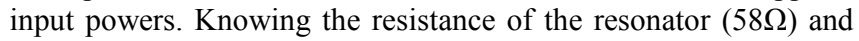
the power output of the network analyzer, the AC current passing through the resonator was calculated for each frequency response (Figure 3b). Figure 3 shows different transmission feedthrough floor levels of $-5.5 \mathrm{~dB}$ and $-3.7 \mathrm{~dB}$ for the conventional and zero bias operation techniques respectively. This change is a result of having different test circuits and consequently different overall impedances between the two ports of the networks analyzer.

It should be noted that in zero bias operation, the only source of power that can thermally actuate the resonator is the AC current (voltage) output the network analyzer. The motional signal for such resonators usually increases by passing more current through them [2]. Therefore, using a highly doped resonator structure can significantly help reduce the electrical resistance of the resonator and increase the current passing through it.

\section{CONCLUSIONS AND FUTURE WORK}

A new operation technique for single crystalline silicon, thermalpiezoresistive resonators was demonstrated that does not require any DC bias current. Due to internal electromechanical mixing, a single AC current is enough to operate the resonator. It is demonstrated that such devices can operate in the electrical domain at half their mechanical resonant frequency without the need for any power supply or biasing components. Therefore, they can potentially be utilized as electronic components without any DC power consumption. In addition, since this technique does not require any supporting electronic components, it further decreases the overall power consumption and circuit foot-print. The demonstrated internal mixing can also be combined with the selfsustained oscillation capability of such resonators to create new opportunities in timekeeping, signal mixing and mass sensing applications.

\section{ACKNOWLEDGEMENTS}

This work was supported by National Science Foundation under grants \#1056068 and \#0923518. 
a)

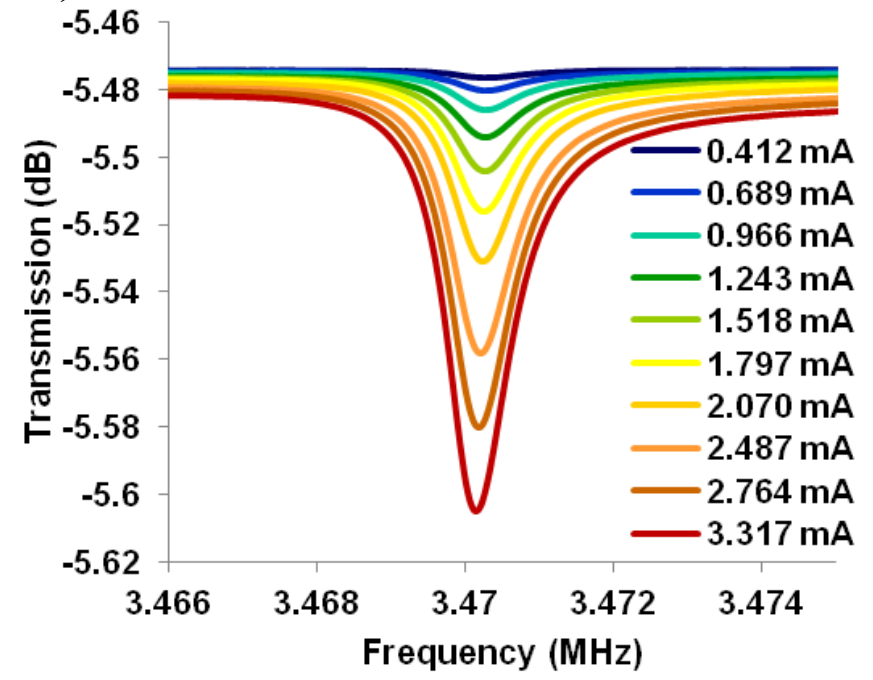

b)

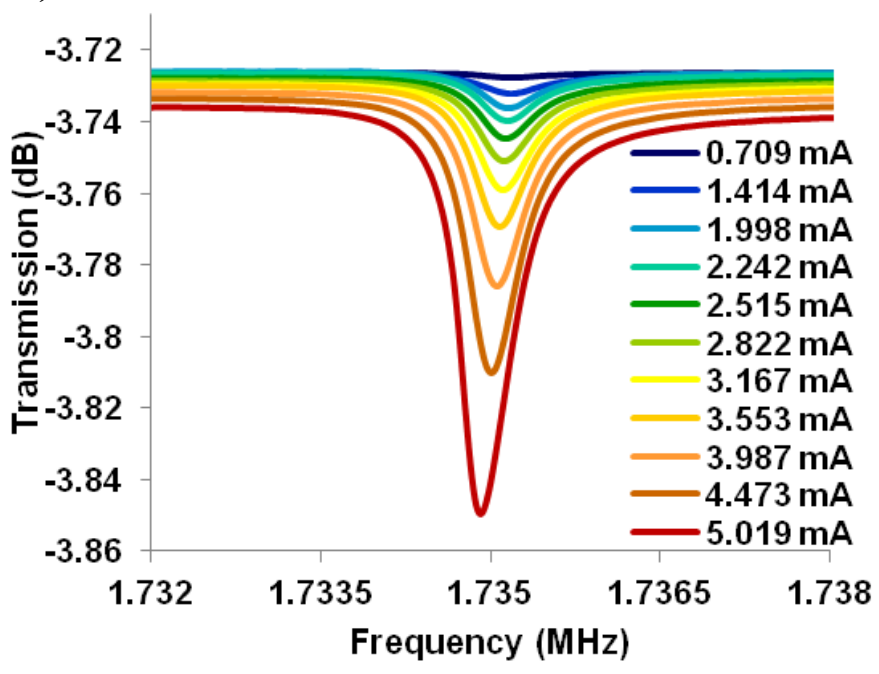

Figure 3: Measured frequency response of the resonator shown in figure 1 directly taken from the network analyzer, a) using the test configuration showed in figure la by application of DC and AC currents at different bias currents (The internal power of the network analyzer was set to $0 \mathrm{~dB}$ which translates into an effective AC current of $2.515 \mathrm{~mA}$ through the resonator), b) using the test configuration shown in figure $1 b$ by applying only an AC signal with different input power levels of -11, -5, -2, -1, 0, 1, 2, 3, 4, 5, and 6 dB that translates into effective AC currents of 0.709 to $5.019 \mathrm{~mA}$ as is shown in the plot. The physical resistance of the resonator is $58 \Omega$. The change in feedthrough level between the two methods is a result of having different testing circuits and eventually different impedances.

\section{REFERENCES}

[1] F. Ayazi, "MEMS for Integrated Timing and Spectral Processing," Invited Paper, Proc. IEEE Custom Integrated Circuits Conference (CICC), pp. 65-72, 2009.

[2] A. Rahafrooz and S. Pourkamali, "High-Frequency Thermally Actuated Electromechanical Resonators With Piezoresistive Readout," IEEE Transactions on Electron Devices, vol.58, no.4, pp.1205-1214, April 2011.

[3] P. G. Steeneken, K. Le Phan, M. J. Goossens, G. E. J. Koops, G. J. A. M. Brom, C. van der Avoort and J. T. M. van Beek, "Piezoresistive heat engine and refrigerator," Natrure physics, Vol. 7, No. 4, pp. 354-350, Apr. 2011.

[4] A. Rahafrooz and S. Pourkamali, "Fully micromechanical piezo-thermal oscillators," 2010 IEEE International Electron Devices Meeting (IEDM), pp.7.2.1-7.2.4, Dec. 2010.

[5] A. Rahafrooz and S. Pourkamali, "Active self-Q-enhancement in high frequency thermally actuated M/NEMS resonators," in Proc. 24rd IEEE MEMS, Cancun, Mexico, Jan, 2011, pp. 760-763.

[6] A. Hajjam, J. C. Wilson, A. Rahafrooz, S. Pourkamali, " Fabrication and characterization of thermally actuated micromechanical resonators for airborne particle mass sensing: II. Device fabrication and characterization," Journal of Micromechanics and Microengineering, vol.20, no.12, Article Number: 125019, Dec. 2010.

[7] J. H. Seo and O. Brand, "High Q-Factor In-Plane-Mode Resonant Microsensor Platform for Gaseous/Liquid Environment," JMEMS 2008, Vol. 17, issue 2, pp. 483-493.

[8] I. Bargatin, I. Kozinsky, and M. L. Roukes, "Efficient Electrothermal Actuation of Multiple Modes of Highfrequency Nanoelectromechanical Resonators," Applied Physics Letters, vol. 90, 2007.

\section{CONTACT}

Amir Rahafrooz, 2390 S. York St., Denver, Colorado, USA, Phone: +1-303-871-2471, Email: amir.rahafrooz@du.edu 